\title{
Simple synthesis of highly uniform bilayer-carbon nanocages
}

\author{
D. A. Ziolkowska, ${ }^{\mathrm{a}, \mathrm{b}, \dagger}$ J. S. Dilip Jangam, ${ }^{\mathrm{a}, \dagger}$ G. Rudakov, ${ }^{\mathrm{c}}$ T. Paronyan, ${ }^{\mathrm{d}}$ M. Akhtar, ${ }^{\mathrm{a}, \mathrm{e}}$ G. Sumanasekera ${ }^{\mathrm{a}, \mathrm{e}}$
} and J. B. Jasinski ${ }^{{ }^{*}}$

a. Conn Center for Renewable Energy Research, University of Louisville, Louisville, KY 40292, USA

b. $\quad$ Faculty of Physics, University of Warsaw, Pasteura 5, 02-093 Warsaw, Poland

c. Department of Physics, Perm State University, Perm, Russia

d. ElectroOptics Research Institute \& Nanotechnology Center, University of Louisville, Louisville, KY 40292, USA

e. $\quad$ Department of Physics and Astronomy, University of Louisville, Louisville, KY 40292, USA

$\dagger$ D. A. Ziolkowska and J. S. Dilip Jangam contributed equally to this work

\begin{abstract}
A simple, highly scalable method of obtaining densely-packed, three-dimensional structures of interconnected, bilayer, hollow carbon nanocages, is reported. High-quality nanocages with well controlled wall thickness are synthesized via catalytic templating on densely-packed, mono-sized nickel nanoparticles, nucleating in situ during short, mid-temperature annealing of an inexpensive precursor obtained from nickel acetate and citric acid. Following nickel dissolution, large monolithic grains of densely packed networks of carbon nanocages are obtained and extensively characterized. In situ TEM experiments are conducted to elucidate the mechanism of nanostructure formation, and a geometrical model is proposed to explain the high specific surface area and its relation to the nanocages' diameter and wall thickness. These nanocages, with their bilayer structure, unimodal pore size distribution and pore size of $\sim 2.5 \mathrm{~nm}$, approach the theoretical capacity of undoped bilayer graphene when tested in an electrochemical double-layer capacitor system. Our synthesis approach provides a facile method for tuning nanocages' morphology and efficiently doping them, creating an effective enhancement of capacity and properties suitable for a broad range of new energy and environmental applications.
\end{abstract}

\footnotetext{
*E-mail: jacek.jasinski@louisville.edu

Tel: +1-502-852-6338
} 


\section{Introduction}

Carbon, with its unrivaled ability to form different hybridization states, can exist in various allotropes, many of which show unique, often drastically different, sets of properties. Diamond and graphite are the best examples: diamond consists of tetrahedrally-coordinated $\mathrm{sp}^{3}$ atoms, each covalently-bonded with its four nearest neighbors, and graphite of a highly anisotropic layer structure of honeycombed atomic planes of strongly-bonded $\mathrm{sp}^{2}$ atoms, with weak $\pi$-type interlayer bonding. Among carbon allotropes, there are also: graphene - a single layer of graphite, and fullerenes - carbons at the nanoscale (whose family includes hollow spheres, ellipsoids, or single- and multi-wall nanotubes) [1]. Carbon nanocages (CNCs) are a particular type of carbon nanoshell, often interconnected and forming three-dimensional (3D) mesoporous structures with significant pore volume and high specific surface area. Recently, they have attracted notable attention due to their unique properties and prospects for applications in gas separation and storage [2, 3], catalysts [4] and catalysts supports [5], as well as electrode materials for energy storage devices [6-8]. CNCs are particularly promising electrode materials for supercapacitors [6, 7] - electrochemical storage appliances which exhibit high power capabilities and play a key role in several technologies [9, 10], including electric transportation, energy management systems [11], and intelligent wireless sensor systems [10]. CNCs show comparable performance to other

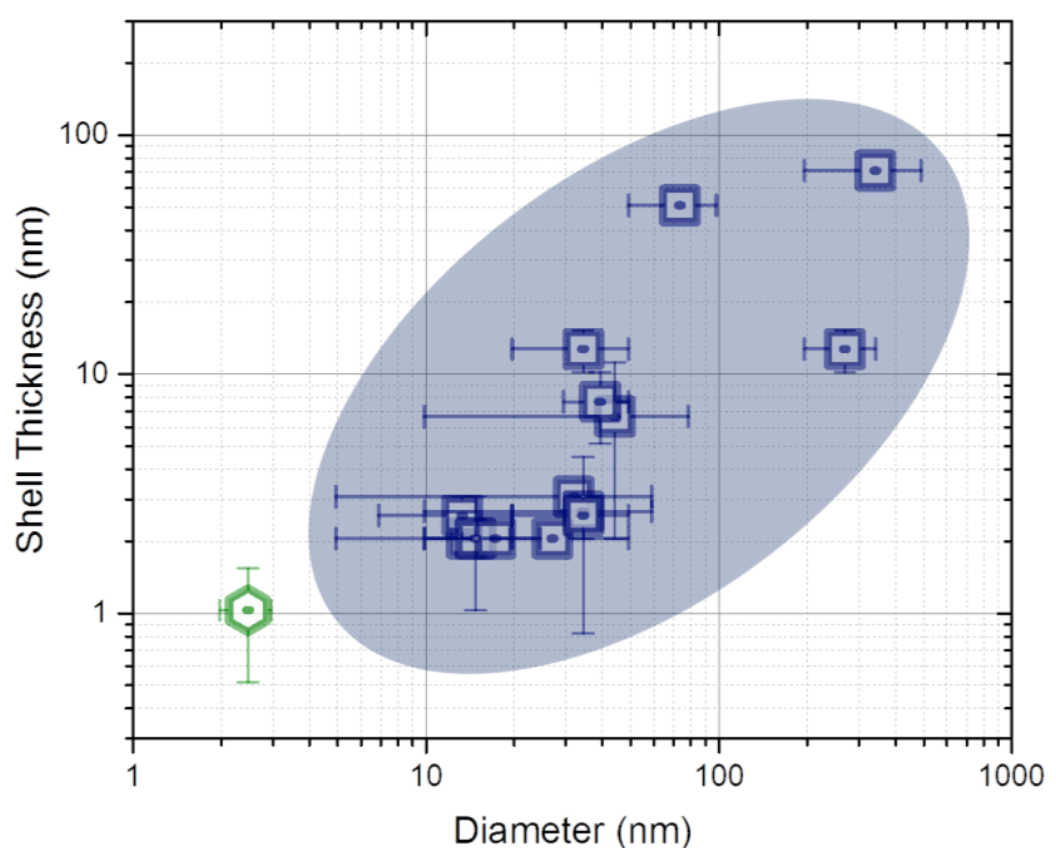

Fig. 1. A comparison of morphological parameters (diameter and shell thickness) of our CNCs (green hexagon) and CNCs reported in literature, obtained using other methods (blue squares) [After Refs 5, 7-9, 11, 21-30]. 
carbons used for supercapacitors [10], such as carbon nanotubes [12], activated carbon (AC) [13, 14], mesoporous carbon [15, 16], and graphene [17-19]. Because of their high specific surface area, CNCs are suitable for electrochemical double layer capacitors (EDLCs), which use an energy storage mechanism based on the physical adsorption of charges at the electrode-electrolyte interface. Doping and adding a pseudo-capacity storage mechanism can further enhance the overall capacity.

Several methods have been proposed to produce CNCs; Ma et al. [20] has used laser-induction complex heating evaporation to create vapors of iron species to react them with methane and form iron nanoparticles coated with graphitic shells, Li et al. [21] have produced CNCs by the deposition of p-xylene over $\mathrm{MgO}$-supported $\mathrm{Co} / \mathrm{Mo}$ catalyst in supercritical $\mathrm{CO}_{2}$, whereas Tsai et al. [22] have used the chelation of the $\mathrm{Cu}^{2+}-\beta$-cyclodextrin complexes by carbonization at $573 \mathrm{~K}$. Some studies [7, 8, 23] have also reported the use of a closed-system approach, with a precursor solution sealed inside a high-pressure autoclave and annealed at high temperature for several hours. Also recently, Wang et al. [5] have used spray pyrolysis of iron carbonyl and carbon precursors. However, the obtained CNCs showed broad size distribution and inhomogeneity. Most of the previous methods pose other challenges, such as long synthesis times, the use of expensive chemicals, scalability issues, or poor size and uniformity control, especially for small CNCs. Here, we report an alternative method that addresses most of these matters. In this approach, short duration synthesis, carried out at moderate temperatures, converts an inexpensive precursor into highly homogenous, densely-packed, interconnected bilayer CNCs. The approach is easily scalable and produces smaller CNCs than any other previously reported [5-8, 10, 20-29], as compared in Figure 1. The highly-uniform, monomodal, 3D mesoporous structure of our CNCs makes this material attractive for applications as electrodes, adsorbents or membranes. Herein, we focus first on our CNC synthesis method. Systematic synthesis studies, using both in situ and ex situ experiments, are conducted, and the formation mechanism is discussed in detail. Samples are synthesized under various conditions and thoroughly studied regarding their morphology, structure, and composition. A proposed geometrical model gives the correlation with their morphological parameters. Finally, the potential of these CNCs for energy applications is demonstrated by preparing EDLCs with them, and measuring their electrochemical performance (which is comparable to undoped bilayer graphene).

\section{Experimental Section}




\subsection{Preparation of Carbon Nanocages}

CNCs are synthesized by a novel in situ nickel template method. Initially, nickel acetate $\left(\mathrm{Ni}\left(\mathrm{CH}_{3} \mathrm{CO}_{2}\right)_{2} \cdot 4 \mathrm{H}_{2} \mathrm{O}\right)$ and citric acid $\left(\mathrm{C}_{6} \mathrm{H}_{8} \mathrm{O}_{7}\right)$ (Sigma-Aldrich) are dissolved in equal proportions (by wt \%) in water. The solution is then dried in an oven at $80{ }^{\circ} \mathrm{C}$, which leaves the precursor powder used for the synthesis of CNCs. The precursor powder, placed in a quartz crucible, is inserted into a horizontal tubular furnace and annealed in a continuous $\mathrm{N}_{2}$ flow at a rate of $80 \mathrm{~cm}^{3} \mathrm{~min}^{-1}$. Five different materials are synthesized by heating the precursor at $50{ }^{\circ} \mathrm{C} \mathrm{min}{ }^{-1}$ from room temperature to $500^{\circ} \mathrm{C}, 550{ }^{\circ} \mathrm{C}, 600^{\circ} \mathrm{C}, 700^{\circ} \mathrm{C}$ and $850{ }^{\circ} \mathrm{C}$, respectively; annealing takes place for $10 \mathrm{~min}$ followed by a cooling period inside the furnace to room temperature, to avoid oxidation. The synthesized powders consist of dense structures of CNCs encapsulating nickel nanoparticles, which are treated with a dilute nitric acid solution to remove nickel, then washed with ethanol and distilled water.

\section{2. $\quad$ Structural characterization and analysis}

The microstructure and morphologies of the CNC samples are investigated using scanning electron microscopy (SEM) in a Carl Zeiss FE-SEM Supra 35VP and transmission electron microscopy (TEM) in a FEI Tecnai F20 operated at $200 \mathrm{kV}$. Several TEM-based techniques, including diffraction contrast imaging, high-resolution transmission electron microscopy (HRTEM), scanning transmission electron microscopy (STEM) and selected area electron diffraction (SAED) are employed to analyze these samples. Nucleation and structural evolution of nickel nanoparticles, as well as the formation of CNCs, are studied in situ in TEM using a singletilt Gatan heating stage. TEM specimens are prepared by dispersing the precursor powder onto gold grids-supported holey carbon films. During in situ heating experiments, specimens are heated at a rate of $50{ }^{\circ} \mathrm{C} \mathrm{min}^{-1}$ to target temperatures $\left(400^{\circ} \mathrm{C}, 500^{\circ} \mathrm{C}, 600^{\circ} \mathrm{C}, 850{ }^{\circ} \mathrm{C}\right)$ and held for $10 \mathrm{~min}$. In addition to SAED measurements, the phase composition and crystal structure are also

analyzed using X-ray diffraction (Bruker D8 Discover) with nickel-filtered $\mathrm{Cu}-\mathrm{K} \alpha$ radiation $(\lambda=1.5418 \AA)$. The Brunauer-Emmett-Teller (BET) specific surface area is determined from nitrogen adsorption-desorption isotherms using a Micromeritics TriStar 3000 system. Two standard materials, alumina $\left(\sim 215 \mathrm{~m}^{2} / \mathrm{g}\right)$ and activated carbon (AC) $\left(\sim 1200 \mathrm{~m}^{2} / \mathrm{g}\right)$, were used to test the system and specific surface area values comparable to nominal ones were obtained.

\subsection{Electrochemical characterization}


Electrochemical measurements are carried out for use in a supercapacitor. Both electrodes are made using $85 \mathrm{wt} \%$ of active material (carbon nanocages synthesized at $600{ }^{\circ} \mathrm{C}$, after the removal of nickel nanoparticles), $10 \%$ of carbon black (CB), and 5\% of polytetrafluoroethylene (PTFE) binder distributed in ethanol. This paste, well-mixed, is coated onto nickel foam current collectors (a surface area of $\sim 0.79 \mathrm{~cm}^{2}$ ) and dried at $120{ }^{\circ} \mathrm{C}$ for 12 hours under vacuum. Electrochemical performance is measured in a symmetric Swagelok ${ }^{\circledR}$ cell configuration system using a cellulose separator and $6 \mathrm{M} \mathrm{KOH}$ aqueous solution as the electrolyte. The charge/discharge measurements are carried out over a potential range between 0 and $0.9 \mathrm{~V}$ in a Pine Research Instrumentation WaveDriver 20 Bipotentiostat/Galvanostat System.
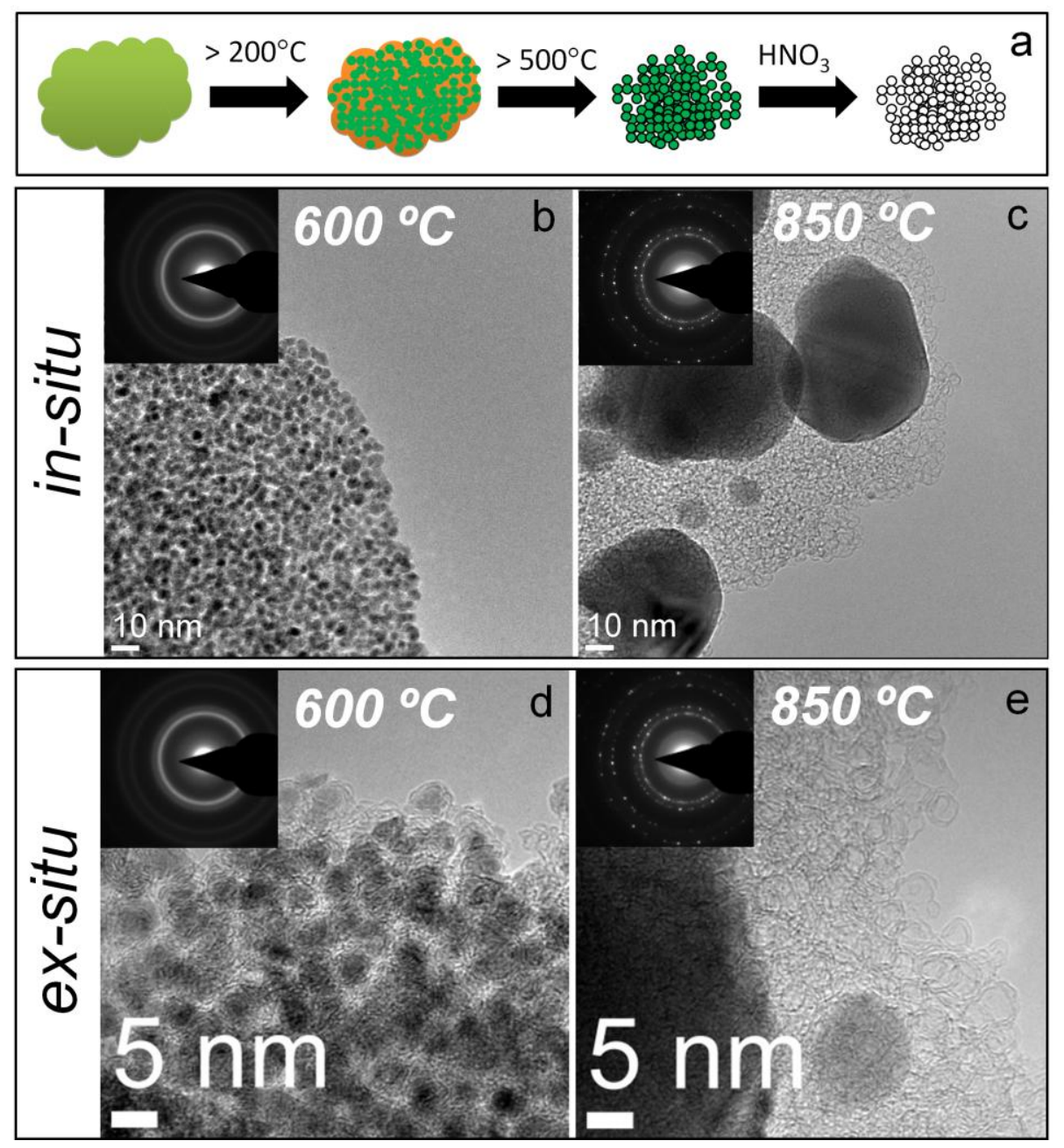

Fig. 2. (a) A schematic diagram showing the synthesis steps. (b-c) TEM images of samples synthesized in situ taken at $600^{\circ} \mathrm{C}$ and $850^{\circ} \mathrm{C}$. (d-e) TEM images of samples synthesized ex situ at $600^{\circ} \mathrm{C}$ and $850^{\circ} \mathrm{C}$, respetively. SAED patterns of measured areas are shown in insets. 


\section{Results and Discussion}

\subsection{CNCs formation mechanism}

3.1.1. In situ studies. As shown schematically in Figure 2a, the synthesis of CNCs consists of the following steps: (1) in situ formation of nickel nanoparticles, (2) catalytic growth of graphitic shells, and (3) removal of nickel nanoparticles through dissolution in $\mathrm{HNO}_{3}$. In situ TEM heating experiments are carried out to clarify the synthesis mechanism and its temperature dependence (Fig. 2b-c). Initially, at a temperature slightly above $250^{\circ} \mathrm{C}$, nickel acetate decomposes to an intermediate phase of nickel carbide $\left(\mathrm{Ni}_{3} \mathrm{C}\right)$ [30], then to nickel metal, followed by a homogenous nucleation of nickel particles. Upon further heating, the so-called dissolution-precipitation mechanism causes a graphitic shell formation [31]. Above $153{ }^{\circ} \mathrm{C}$ [32], citric acid melts and undergoes dehydration and decarboxylation leading to the formation of intermediate cyclic organic compounds, which then decompose upon continued heating [33]. The amorphous carbon formed in this process gradually begins to dissolve into nickel particles. Upon reaching supersaturation, at temperatures between $500{ }^{\circ} \mathrm{C}$ and $600^{\circ} \mathrm{C}$, it starts to segregate on the surface of nickel particles [34] leading to the formation of a 3D network of thin interconnected graphitic nanoshells (i.e., CNCs), each encapsulating a nickel particle. Nickel is an efficient catalyst for the graphitization of amorphous carbon; its high catalytic activity originates from the d-electron configuration and ionization potential [31]. The formation of graphitic shells at relatively low temperatures observed in our samples (starting at $\sim 500^{\circ} \mathrm{C}$ ), is due to the small size of nickel particles and enhanced carbon diffusion. Upon further heating above $\sim 600^{\circ} \mathrm{C}$, Ostwald ripening occurs through the melting of smaller Ni particles, and the re-attaching of released nickel atoms to larger ones leaving the carbon shells mostly intact (Fig. 2c). This phenomena leads to a reduction of total surface area of $\mathrm{Ni}$ particles and their energy [35]. This happens at a much lower temperature than the $1455^{\circ} \mathrm{C}$ melting point of bulk nickel, due to the nanoscale size of the particles [36].

3.1.2. Ex situ studies. CNCs are also synthesized ex situ in a furnace. Similarly to in situ experiments, anenaling in temperatures between $500^{\circ} \mathrm{C}$ and $600{ }^{\circ} \mathrm{C}$ leads to the formation of homogenous, densely-packed, mostly bilayer $\sim 3 \mathrm{~nm}$ carbon nanocages, each encapsulating a $\mathrm{Ni}$ particle, as shown in Fig 2d (TEM image of a typical sample synthesized at $600{ }^{\circ} \mathrm{C}$ ). Similarly to in situ experiments, Ostwald ripening takes place during annealing at higher temperatures, as evidenced by data from samples annealed at $850^{\circ} \mathrm{C}$ (see an example TEM image in Fig. 2e). In these samples, Ni out-diffused from their small $\sim 3 \mathrm{~nm}$ carbon shells and agglomerated in the form 

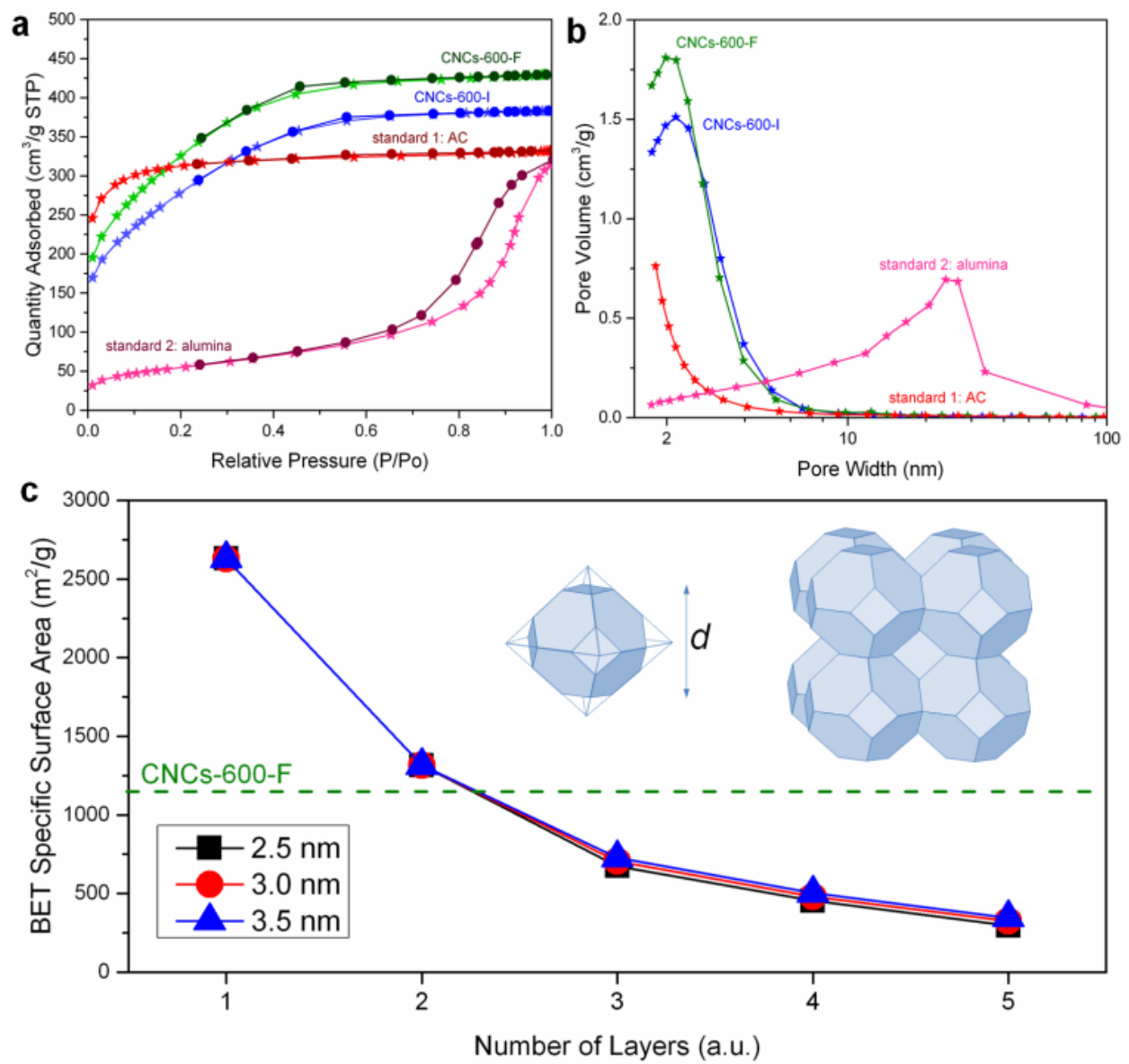

Fig. 3. (a) $\mathrm{N}_{2}$ adsorption (stars) and desorption (circles) isotherms measured using the BET method for CNC samples synthesized at $600^{\circ} \mathrm{C}$ (after partial (CNCs-600-I) and almost complete (CNCs-600-F) nickel removal) and two standard samples - AC and alumina. (b) Corresponding BET-based pore size distribution plots. (c) BET specific surface area calculated using the truncated octahedron model for three nanocage diameters and shell thickness from 1 to 5 carbon layers. The dashed line represents the experimental value of $\sim 1150$ $\mathrm{m}^{2} / \mathrm{g}$ measured for the $600^{\circ} \mathrm{C} \mathrm{CNCs}$.

of large Ni particles. As in the case of in situ annealing, this mechanism leads to the formation of empty CNCs. Figure S1, Supplementary Data, shows additional TEM results on the effect of temperature.

Morphological changes occurring during annealing are also reflected in SAED patterns; Continuous diffraction rings observed at lower temperatures (Fig. 2b-c-inset) are replaced by the strong spotty rings of large particles formed at high temperatures (Fig. 2d-e-insets). SAED analysis (Fig. S2, Supplementary Data) also confirms the metallic phase of nickel (ICDD PDF card No. 00-001-1258. 
Following synthesis experiments, which demonstrate the formation of CNCs above $\sim 500{ }^{\circ} \mathrm{C}$ and Oswald ripening above $\sim 600^{\circ} \mathrm{C}$, the material synthesized at $600^{\circ} \mathrm{C}$ is expected to have a stable structure and uniform morphology compared to samples synthesized at lower or higher temperatures. Therefore, the material synthesized at $600{ }^{\circ} \mathrm{C}$ is selected for further study, including the preparation of CNCs, fabrication of supercapacitor electrodes, and electrochemical testing. Purified CNCs are obtained from the $600{ }^{\circ} \mathrm{C}$ material by removal of nickel through dissolution in $\mathrm{HNO}_{3}$ (the advantage of this approach is the possibility of recovering and reusing $\mathrm{Ni}$ ions from the solvent solution, which is potentially more sustainable and environmentally friendly). The material is thoroughly characterized and tested as discussed in the following.
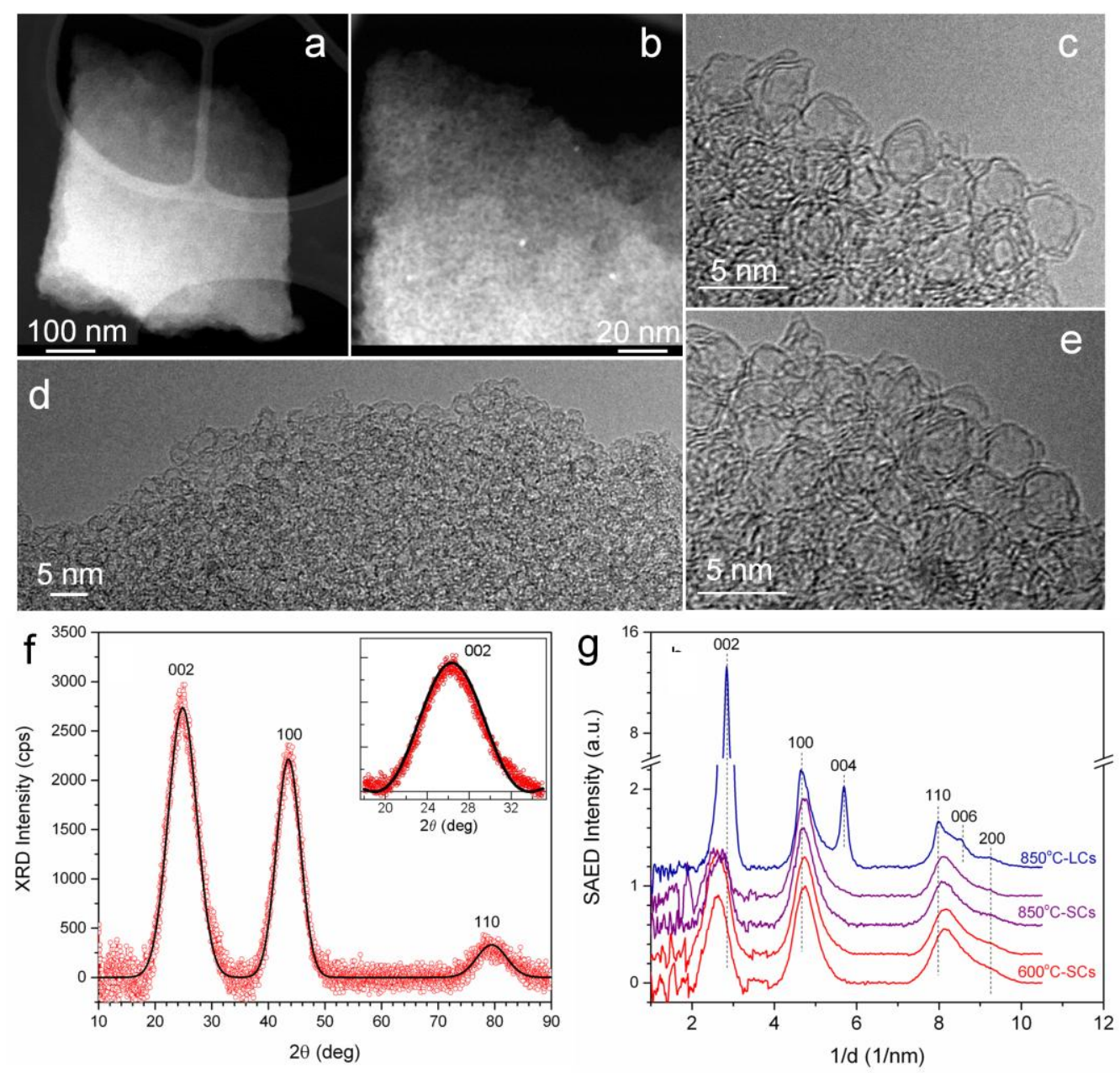

Fig. 4. STEM image of a small grain of CNC sample synthesized at $600^{\circ} \mathrm{C}$ examined at (a) lower and (b) higher magnifications. (c) Low- and (d-e) high-magnification HRTEM images of several CNCs at the edge of a sample grain (scale bars show $5 \mathrm{~nm}$ ). (f) XRD pattern of the material synthesized at $600^{\circ} \mathrm{C}$ (without nickel) after subtracting the background. Inset figure shows an experimental XRD profile (red circles) and the fitted curve (black solid line) - see Supplementary Data . (g) SAED intensity profiles for the sample synthesized at $600^{\circ} \mathrm{C}$ (red) and $850^{\circ} \mathrm{C}$ (violet and blue) after nickel removal; SAED patterns measured on $850^{\circ} \mathrm{C}$ sample show differences between small cages (SCs) (violet) and large cages (LCs) (blue) profiles; All profiles are normalized at the maximum of the (100) peak and are offset vertically for clarity. 


\subsection{Characterization and properties of CNCs after Ni removal}

3.2.1. Porosity. The monolithic structure of the microporous carbon is confirmed by SEM (Fig. S3, Supplementary Data) and its porosity is analyzed by BET method (Fig. 3a). The BET-derived specific surface area values measured for CNC samples with fully and partially removed nickel are $\sim 1150 \mathrm{~m}^{2} / \mathrm{g}$ and $990 \mathrm{~m}^{2} / \mathrm{g}$, respectively. The analysis shows a high specific surface area and a monomodal pore size distribution centered at $\sim 2.5 \mathrm{~nm}$ (Fig. 3b). A geometrical model of the 3D network of nanocages based on densely-packed truncated octahedrons (Fig. S4, Supplementary Data), which are the only known space-filling polyhedrons beside cubes, is proposed. The results, i.e., specific surface area calculated for several nanocage diameters and a different number of layers, are shown in Figure 3c and compared with the experimental value measured from BET. This analysis indicates two or occasionally three atomic layers in the nanocage shell, which is in excellent agreement with HRTEM and XRD, as discussed in the following.

Z-contrast STEM and HRTEM imaging are also used to image the nanoscale porosity of fabricated materials; Z-contrast images (Fig. 4a-b) show large monolithic grains of mesoporous CNCs material with densely-packed mesopores, each in the 2-3 nm size range. HRTEM images (Fig. 4c-e) indicates that the mesopores are interconnected nanocages with bilayer shells.

3.2.2. Shell thickness. A typical XRD pattern of material synthesized at $600{ }^{\circ} \mathrm{C}$, after background subtraction, is shown in Figure 4f. The pattern exhibits relatively broad (002), (100) and (110) peaks, located at $24.84^{\circ}, 43.55^{\circ}$ and $79.46^{\circ}$, i.e., at the $\mathrm{d}$-spacing values of $3.58,2.08$ and $1.21 \AA$, respectively. While the (002) d-spacing is significantly larger than the $3.3555 \AA$ in graphite (which may be due to some oxygen presence and the fact that only a weak $\pi$-type bonding connects carbon layers to their neighbors), the (100) and (110)d-spacing values are slightly smaller than $2.13389 \AA$ and $1.23200 \AA$ values for graphite. However, these planes are associated with a strong in-plane $\mathrm{sp}^{2}$-bonding and are therefore less susceptible to any changes. The observed slight reduction of their d-spacing values is probably due to the significant carbon layer bending caused by the large curvature of the nanocages.

Electron diffraction is also used for crystal structure analysis. Figure $4 \mathrm{~g}$ shows a comparison of SAED intensity profiles from CNCs synthesized at $600{ }^{\circ} \mathrm{C}$, and $850{ }^{\circ} \mathrm{C}$. For the $600{ }^{\circ} \mathrm{C}$ sample, the (002), (100), (110) and weak (200) peaks are observed at d-spacing values of $3.82 \AA, 2.12 \AA$, $1.23 \AA$, and $1.08 \AA$, respectively. Similarly to XRD, in-plane d-spacing values are much closer (within $1 \%$ ) to graphite, whereas the spacing between layers is significantly larger than the one of 
graphite. For the $850{ }^{\circ} \mathrm{C}$ sample, two different types of SAED patterns are found, in agreement with two different morphologies observed in TEM. For small cages (SCs) $(\sim 3 \mathrm{~nm})$, patterns are similar to those observed for the $600^{\circ} \mathrm{C}$ sample, whereas patterns measured for large cages (LCs) ( 20-100 nm) are typical of multilayer graphitic structures; The (004) and (006) rings appear in addition to the rings observed for SCs, and the (002) ring is much stronger. The d-spacing values of $3.51 \AA, 2.15 \AA, 1.76 \AA, 1.25 \AA, 1.17 \AA$ and $1.08 \AA$, are found for LCs. That means that values for the $(00 l)$-type planes are on average $4.5 \pm 0.3 \%$ larger, whereas the ones for in-plane planes are only about $1.2 \pm 0.6 \%$ greater than those in graphite. A highly anisotropic bonding strength can explain this change. SAED results in Figure $4 \mathrm{~g}$ shows significantly higher crystallinity of LCs, compared to SCs. This crystallinity improvement is evidenced by the appearance of the (004) and (006) diffraction rings, as well as by significant narrowing of all rings. These results agree with the HRTEM study.

Carbon structures of CNC samples are also analyzed using Raman spectroscopy (for details see Supplementary Data). The shape of the Raman spectra is similar to the one reported for MWCNTs and seems to originate from the large curvature of the nanocages $[37,38]$. 

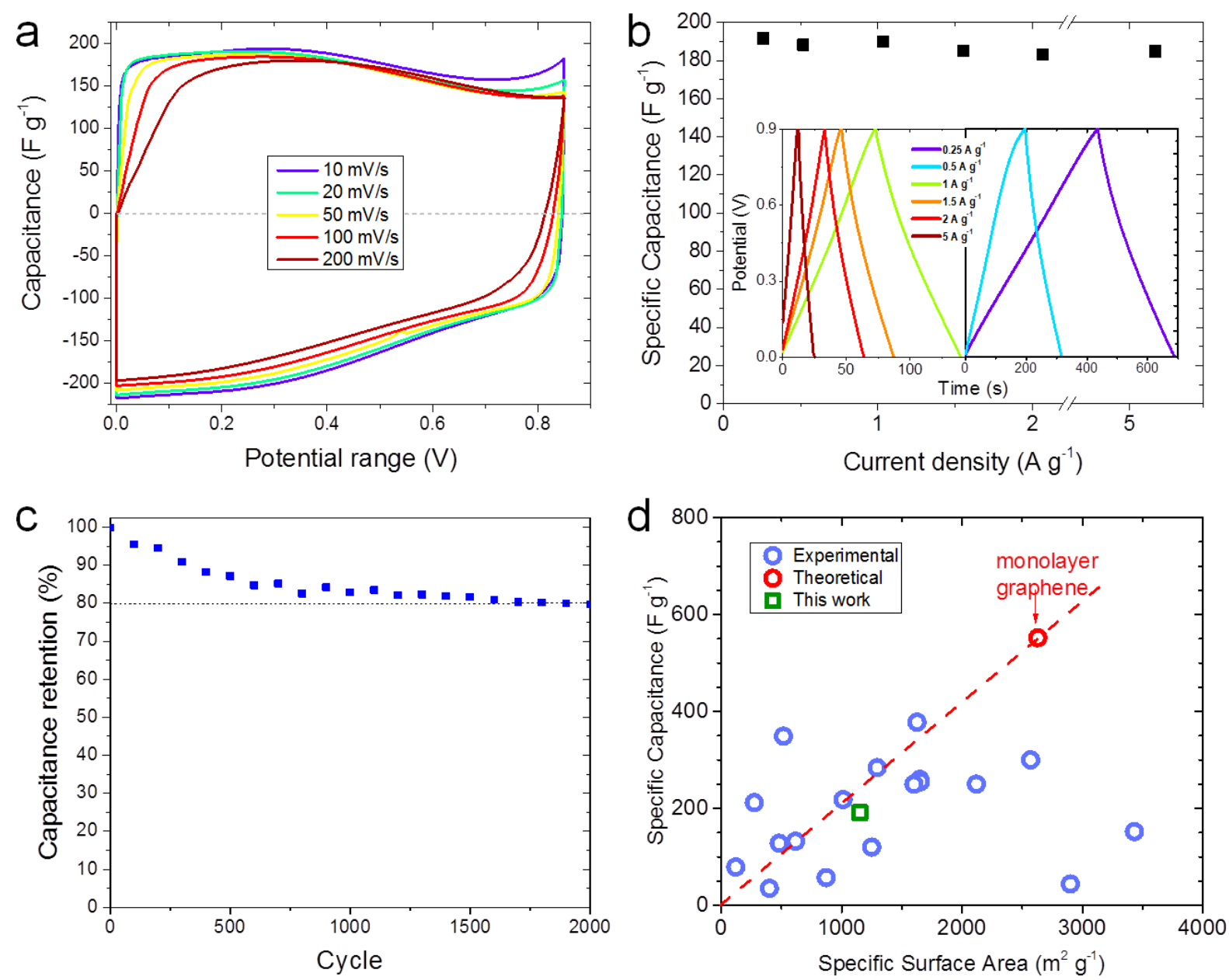

Fig. 5. (a) Cycling voltammetry curves measured at scan rate 10 to $200 \mathrm{mV} / \mathrm{s}$. (b) Specific capacitance of CNCs supercapacitor and (inset) corresponding galvanostatic charge-discharge curves at current densities between $250 \mathrm{~mA} \mathrm{~g}^{-1}$ and

\subsection{Applications of CNCs - EDLC example}

Electrochemical measurements are conducted for the use of nanocages as an electrode material in supercapacitors. The electrodes are prepared from the material synthesized at $600{ }^{\circ} \mathrm{C}$ after nickel removal. Figure 5 shows preliminary results of EDLC supercapacitor characteristics with carbon nanocages comprising the active material of both electrodes. The $\mathrm{CV}$ curves recorded at the scan rate between 10 to $200 \mathrm{mV} \mathrm{s}^{-1}$ are nearly rectangular, even at a high scan rate, which proves welldeveloped capacitance properties with rapid diffusion, and the easy transport of electrolyte ions to the interface with the electrode. The small distortion of the ideal capacitor shape is due to the 
presence of functional groups responsible for pseudocapacitance [39]. When the full cell is charged and discharged between 0 and $0.9 \mathrm{~V}$ at current densities between $250 \mathrm{~m} \mathrm{~A} \mathrm{~g}^{-1}$ and $5 \mathrm{Ag} \mathrm{g}^{-1}$ the specific capacitance remains stable, which is typical for mesoporous materials. The observed almost linear voltage vs. time relation is typical for an electric double layer capacitance. There is a slight distortion of the curves, presumably caused by the pseudo-capacitance of functional groups. The XPS analysis of funtional groups present in the material is disucussed in Supporting Information (Fig. S6). The molar content of oxygen is estimated at $15 \%$ in this material. The specific capacitance of one electrode $\left(\mathrm{C}_{\mathrm{sp}}\right)$ is calculated by Equation 1 [7, 40]:

$$
C_{s p}=4 \times \frac{I \Delta t}{m \Delta V}
$$

where $I, \Delta t, m$, and $\Delta V$ are the applied current, the discharge time, the mass of the active material on two electrodes, and the voltage range, respectively. First cycle capacity of $\sim 190 \mathrm{~F} \mathrm{~g}^{-1}$ stabilizes at $\sim 160 \mathrm{Fg}^{-1}$ for over 700 cycles at the current density $1 \mathrm{~A} \mathrm{~g}^{-1}$. The long cycling test shows that the device maintains $\sim 80 \%$ of its initial capacity after 2000 cycles at $1 \mathrm{~A} \mathrm{~g}^{-1}$ current density (Fig. $5 \mathrm{c})$. The specific capacitance of $190 \mathrm{~F} \mathrm{~g}^{-1}$ is comparable with values reported for undoped carbonbased material [6, 7, 41, 42]. A recent review of supercapacitor carbon electrodes [10] ranks numerous carbons based on their specific capacity values. Although some materials exhibit values higher than $\sim 300 \mathrm{~F} \mathrm{~g}^{-1}$, most of them have either a complicated nanocomposite structure requiring extensive fabrication methods, or their high values originate from doping (e.g., nitrogen, fluorine, etc.) and the pseudo-capacity mechanism [10]. This includes biomass-derived materials [43]. The method of gravimetric capacitance determination must be considered when comparing values, since a three-electrode set-up tends to produce significantly higher values [44, 45]. Practical systems are closely related to the two-electrode cell configuration, with which we characterize our material.

The high double-layer capacitance of our material is evaluated by a comparison to other undoped graphene-like carbons with similar specific surface areas (SSA). The measured double-layer capacitance on one side of a graphene layer is estimated at $21 \mu \mathrm{F} \mathrm{cm}{ }^{-2}$ [1], and its theoretical SSA (total from both sides) is $2630 \mathrm{~m}^{2} \mathrm{~g}^{-1}$. If that is the case, the gravimetric specific double-layer capacitance of monolayer graphene is expected at about $550 \mathrm{~F} \mathrm{~g}^{-1}$. In a simple estimation, a double-layer capacitance of undoped graphene-like structures should relate to this value by a simple factor of specific surface area ratio between the material and monolayer graphene. Our 
CNCs follow that trend and almost reach the theoretical value for a bilayer graphene as shown in Fig. 5d [10] (slightly lower than expected capacity because some CNCs have tri-layer shells).

The quality of our material and our level of control over wall thickness is undoubtedly better than in previously reported nanospherical carbons [5-8, 10, 20-29]. With their bilayer structure, unimodal pore size distribution, and pore size of $\sim 2.5 \mathrm{~nm}$, the material approaches the theoretical capacity of bilayer graphene. The fabrication of CNCs with controllable pore size might play an important role in capacitance control by preventing pore saturation and surface accessibility problems, which is the main cause of underperformance in typical high surface area carbons (see Fig. 5d). Our method should allow for tuning of CNC diameters and shell thicknesses by controlling precursor composition and synthesis temperature. Due to its simplicity, the method will also enable easy in situ doping, directly during the synthesis step; our current efforts are focused on obtaining nitrogen-doped CNCs and adding a large pseudo-capacity. Our easy synthesis approach provides new opportunities for additional electrochemical applications, including electrodes for lithium-ion, or lithium-sulfur batteries, catalyst supports for heterogeneous catalysts, or in the case of doped CNCs, electrocatalysts for energy-relevant processes such as hydrogen evolution or oxygen reduction reactions [8, 46-48].

\section{Conclusions}

We have demonstrated a simple, scalable method of producing bulk quantities of the smallest CNCs ever reported, organized into monolithic, homogenous, densely-packed 3D structures, with significant potential applications in energy and environmental technologies. Our method involves a short annealing of an inexpensive precursor based on $\mathrm{Ni}$ acetate and citric acid, and creates CNCs with mostly bilayer shells (CNCs with tri-layer shells are also formed, occasionally). They exhibit a high specific surface area, which is described as a function of nanocage parameters, using a geometrical model of densely-packed truncated octahedrons. The CNCs show high double-layer capacitance in EDLC structures, approaching that of bilayer graphene. A significant increase of capacity is expected through nanostructure optimization and efficient doping using our synthesis approach. This method represents a scalable alternative to produce bilayer 3D CNCs in large quantities for use in energy and environmental applications. 


\section{Acknowledgements}

The authors acknowledge the Conn Center for Renewable Energy Research and funding from the US National Science Foundation EPSCoR Program (sub-award no. 3048111570-15-016). DAZ also acknowledges the support from the Mobility Plus Program funded by the Ministry of Science and Higher Education of the Republic of Poland. The authors would like to thank Dr. Anna A. Latoszynska for insightful discussions concerning testing of supercapacitor structures.

\section{Supplementary Data}

Supplementary Data is available.

\section{References:}

[1] A. Aqel, K.M.A. El-Nour, R.A. Ammar, A. Al-Warthan, Carbon nanotubes, science and technology part (I) structure, synthesis and characterisation, Arabian Journal of Chemistry 5(1) (2012) 1-23.

[2] W. Wang, D. Yuan, Mesoporous carbon originated from non-permanent porous MOFs for gas storage and CO2/CH4 separation, Scientific Reports 4 (2014) 5711.

[3] W. Xia, B. Qiu, D. Xia, R. Zou, Facile preparation of hierarchically porous carbons from metal-organic gels and their application in energy storage, Scientific Reports 3 (2013) 1935.

[4] S. Chen, J.Y. Bi, Y. Zhao, L.J. Yang, C. Zhang, Y.W. Ma, Q. Wu, X.Z. Wang, Z. Hu, Nitrogen-Doped Carbon Nanocages as Efficient Metal-Free Electrocatalysts for Oxygen Reduction Reaction, Advanced Materials 24(41) (2012) 5593-5597.

[5] X.X. Wang, Z.H. Tan, M. Zeng, J.N. Wang, Carbon nanocages: A new support material for Pt catalyst with remarkably high durability, Scientific Reports 4 (2014) 4437.

[6] K. Xie, X.T. Qin, X.Z. Wang, Y.N. Wang, H.S. Tao, Q. Wu, L.J. Yang, Z. Hu, Carbon Nanocages as Supercapacitor Electrode Materials, Advanced Materials 24(3) (2012) 347-352.

[7] Y. Tan, C. Xu, G. Chen, Z. Liu, M. Ma, Q. Xie, N. Zheng, S. Yao, Synthesis of ultrathin nitrogen-doped graphitic carbon nanocages as advanced electrode materials for supercapacitor, ACS Applied Materials \& Interfaces 5(6) (2013) 2241-8.

[8] G. Li, L. Xu, Q. Hao, M. Wang, Y. Qian, Synthesis, characterization and application of carbon nanocages as anode materials for high-performance lithium-ion batteries, RSC Advances 2(1) (2012) 284-291.

[9] P. Simon, Y. Gogotsi, Materials for electrochemical capacitors, Nature Materials 7(11) (2008) 845-854.

[10] L.G.H. Staaf, P. Lundgren, P. Enoksson, Present and future supercapacitor carbon electrode materials for improved energy storage used in intelligent wireless sensor systems, Nano Energy 9 (2014) 128-141.

[11] J.R. Miller, P. Simon, Materials science - Electrochemical capacitors for energy management, Science 321(5889) (2008) 651-652.

[12] D.N. Futaba, K. Hata, T. Yamada, T. Hiraoka, Y. Hayamizu, Y. Kakudate, O. Tanaike, H. Hatori, M. Yumura, S. lijima, Shape-engineerable and highly densely packed single-walled carbon nanotubes and their application as super-capacitor electrodes, Nature Materials 5(12) (2006) 987-994.

[13] V. Ruiz, C. Blanco, R. Santamaria, J.M. Ramos-Fernandez, M. Martinez-Escandell, A. Sepulveda-Escribano, F. Rodriguez-Reinoso, An activated carbon monolith as an electrode material for supercapacitors, Carbon $47(1)$ (2009) 195-200.

[14] G. Gryglewicz, J. Machnikowski, E. Lorenc-Grabowska, G. Lota, E. Frackowiak, Effect of pore size distribution of coal-based activated carbons on double layer capacitance, Electrochimica Acta 50(5) (2005) 1197-1206. 
[15] K. Wang, Y. Wang, Y. Wang, E. Hosono, H. Zhou, Mesoporous Carbon Nanofibers for Supercapacitor Application, Journal of Physical Chemistry C 113(3) (2009) 1093-1097.

[16] W. Xing, S.Z. Qiao, R.G. Ding, F. Li, G.Q. Lu, Z.F. Yan, H.M. Cheng, Superior electric double layer capacitors using ordered mesoporous carbons, Carbon 44(2) (2006) 216-224.

[17] Y. Zhu, S. Murali, M.D. Stoller, K.J. Ganesh, W. Cai, P.J. Ferreira, A. Pirkle, R.M. Wallace, K.A. Cychosz, M. Thommes, D. Su, E.A. Stach, R.S. Ruoff, Carbon-Based Supercapacitors Produced by Activation of Graphene, Science 332(6037) (2011) 1537-1541.

[18] M.D. Stoller, S. Park, Y. Zhu, J. An, R.S. Ruoff, Graphene-Based Ultracapacitors, Nano Letters 8(10) (2008) 3498-3502.

[19] Y. Wang, Z. Shi, Y. Huang, Y. Ma, C. Wang, M. Chen, Y. Chen, Supercapacitor Devices Based on Graphene Materials, Journal of Physical Chemistry C 113(30) (2009) 13103-13107.

[20] Y. Ma, Z. Hu, K. Huo, Y. Lu, Y. Hu, Y. Liu, J. Hu, Y. Chen, A practical route to the production of carbon nanocages, Carbon 43(8) (2005) 1667-1672.

[21] Z. Li, M. Jaroniec, P. Papakonstantinou, J.M. Tobin, U. Vohrer, S. Kumar, G. Attard, J.D. Holmes, Supercritical Fluid Growth of Porous Carbon Nanocages, Chemistry of Materials 19(13) (2007) 3349-3354.

[22] C.K. Tsai, H.Y. Kang, C.I. Hong, C.H. Huang, F.C. Chang, H.P. Wang, Preparation of hollow spherical carbon nanocages, Journal of Nanoparticle Research 14(12) (2012).

[23] G. Li, H. Yu, L. Xu, Q. Ma, C. Chen, Q. Hao, Y. Qian, General synthesis of carbon nanocages and their adsorption of toxic compounds from cigarette smoke, Nanoscale 3(8) (2011) 3251-7.

[24] D.M. Burke, J.P. O'Byrne, P.G. Fleming, D. Borah, M.A. Morris, J.D. Holmes, Carbon nanocages as heavy metal ion adsorbents, Desalination 280(1-3) (2011) 87-94.

[25] Y. Li, C. Zhou, X.J. Xie, G.Q. Shi, L.T. Qu, Spontaneous, catalyst-free formation of nitrogen-doped graphitic carbon nanocages, Carbon 48(14) (2010) 4190-4196.

[26] Z.Y. Lyu, D. Xu, L.J. Yang, R.C. Che, R. Feng, J. Zhao, Y. Li, Q. Wu, X.Z. Wang, Z. Hu, Hierarchical carbon nanocages confining high-loading sulfur for high-rate lithium-sulfur batteries, Nano Energy 12 (2015) 657-665.

[27] S.J. Teng, X.X. Wang, B.Y. Xia, J.N. Wang, Preparation of hollow carbon nanocages by iodine-assisted heat treatment, Journal of Power Sources 195(4) (2010) 1065-1070.

[28] R.Y. Zhang, M. Hummelgard, H. Olin, Carbon nanocages grown by gold templating, Carbon 48(2) (2010) 424430.

[29] J. Zhao, H.W. Lai, Z.Y. Lyu, Y.F. Jiang, K. Xie, X.Z. Wang, Q. Wu, L.J. Yang, Z. Jin, Y.W. Ma, J. Liu, Z. Hu, Hydrophilic Hierarchical Nitrogen-Doped Carbon Nanocages for Ultrahigh Supercapacitive Performance, Advanced Materials 27(23) (2015) 3541-3545.

[30] M.A. Mohamed, S.A. Halawy, M.M. Ebrahim, NONISOTHERMAL DECOMPOSITION OF NICKEL ACETATE TETRAHYDRATE, Journal of Analytical and Applied Pyrolysis 27(2) (1993) 109-118.

[31] L. Baraton, Z.B. He, C.S. Lee, C.S. Cojocaru, M. Châtelet, J.-L. Maurice, Y.H. Lee, D. Pribat, On the mechanisms of precipitation of graphene on nickel thin films, EPL (Europhysics Letters) 96(4) (2011) 46003.

[32] M.M. Barbooti, D.A. Alsammerrai, THERMAL-DECOMPOSITION OF CITRIC-ACID, Thermochimica Acta 98 (1986) 119-126.

[33] Y. Narendar, G.L. Messing, Mechanisms of phase separation in gel-based synthesis of multicomponent metal oxides, Catalysis Today 35(3) (1997) 247-268.

[34] A. Oya, S. Otani, CATALYTIC GRAPHITIZATION OF CARBONS BY VARIOUS METALS, Carbon 17(2) (1979) 131137.

[35] A. Baldan, Review progress in Ostwald ripening theories and their applications to nickel-base superalloys Part I: Ostwald ripening theories, Journal of materials science 37(11) (2002) 2171-2202.

[36] B.D. Cullity, S.R. Stock, Elements of X-Ray Diffraction, Third ed., Prentice Hall2001.

[37] G. Grassi, A. Scala, A. Piperno, D. lannazzo, M. Lanza, C. Milone, A. Pistone, S. Galvagno, A facile and ecofriendly functionalization of multiwalled carbon nanotubes by an old mesoionic compound, Chem. Commun. 48(54) (2012) 6836-6838.

[38] P. Delhaes, M. Couzi, M. Trinquecoste, J. Dentzer, H. Hamidou, C. Vix-Guterl, A comparison between Raman spectroscopy and surface characterizations of multiwall carbon nanotubes, Carbon 44(14) (2006) 3005-3013. 
[39] K. Chen, S. Song, D. Xue, Beyond graphene: materials chemistry toward high performance inorganic functional materials, Journal of Materials Chemistry A 3(6) (2015) 2441-2453.

[40] A.A. Latoszynska, G.Z. Zukowska, I.A. Rutkowska, P.-L. Taberna, P. Simon, P.J. Kulesza, W. Wieczorek, Nonaqueous gel polymer electrolyte with phosphoric acid ester and its application for quasi solid-state supercapacitors, Journal of Power Sources 274 (2015) 1147-1154.

[41] L. Qie, W. Chen, H. Xu, X. Xiong, Y. Jiang, F. Zou, X. Hu, Y. Xin, Z. Zhang, Y. Huang, Synthesis of functionalized 3D hierarchical porous carbon for high-performance supercapacitors, Energy \& Environmental Science 6(8) (2013) 2497-2504.

[42] X.Y. Chen, C. Chen, Z.J. Zhang, D.H. Xie, X. Deng, J.W. Liu, Nitrogen-doped porous carbon for supercapacitor with long-term electrochemical stability, Journal of Power Sources 230 (2013) 50-58.

[43] B. Xu, Y. Chen, G. Wei, G. Cao, H. Zhang, Y. Yang, Activated carbon with high capacitance prepared by $\mathrm{NaOH}$ activation for supercapacitors, Materials Chemistry and Physics 124(1) (2010) 504-509.

[44] M.D. Stoller, R.S. Ruoff, Best practice methods for determining an electrode material's performance for ultracapacitors, Energy \& Environmental Science 3(9) (2010) 1294-1301.

[45] V. Khomenko, E. Frackowiak, F. Beguin, Determination of the specific capacitance of conducting polymer/nanotubes composite electrodes using different cell configurations, Electrochimica Acta 50(12) (2005) 2499-2506.

[46] K. Wang, Z. Li, Y. Wang, H. Liu, J. Chen, J. Holmes, H. Zhou, Carbon nanocages with nanographene shell for high-rate lithium ion batteries, Journal of Materials Chemistry 20(43) (2010) 9748-9753.

[47] J. Zhang, C.P. Yang, Y.X. Yin, L.J. Wan, Y.G. Guo, Sulfur Encapsulated in Graphitic Carbon Nanocages for High-Rate and Long-Cycle Lithium-Sulfur Batteries, Advanced Materials 28(43) (2016) 9539-9544.

[48] L. Shen, T. Sun, O. Zhuo, R. Che, D. Li, Y. Ji, Y. Bu, Q. Wu, L. Yang, Q. Chen, Alcohol-tolerant Platinum Electrocatalyst for Oxygen Reduction by Encapsulating Platinum Nanoparticles inside Nitrogen-doped Carbon Nanocages, ACS Applied Materials \& Interfaces (2016). 

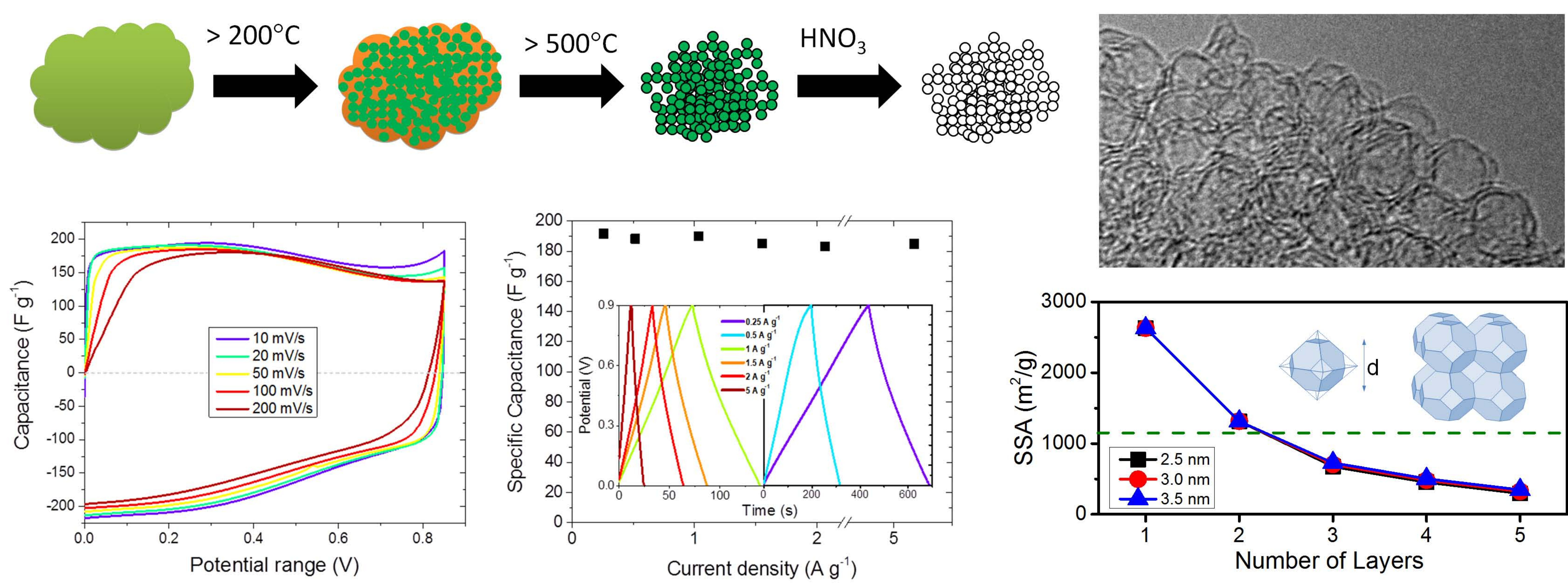